\title{
Investigation of transient conduction-radiation heat transfer in a square cavity using combination of LBM and FVM
}

\author{
MOHAMMAD MEHDI KESHTKAR ${ }^{1, *}$ and POUYAN TALEBIZADEHSARDARI ${ }^{2}$ \\ ${ }^{1}$ Department of Mechanical Engineering, School of Engineering, Kerman Branch, Islamic Azad University, \\ Kerman, Iran \\ ${ }^{2}$ Faculty of Engineering, The University of Nottingham, University Park, Nottingham NG7 2RD, United \\ Kingdom \\ e-mail: mkeshtkar54@yahoo.com
}

MS received 8 April 2017; revised 11 September 2017; accepted 10 November 2017; published online 17 April 2018

\begin{abstract}
In this paper, the effect of surface radiation in a square cavity containing an absorbing, emitting and scattering medium with four heated boundaries is investigated, numerically. Lattice Boltzmann method (LBM) is used to solve the energy equation of a transient conduction-radiation heat transfer problem and the radiative heat transfer equation is solved using finite-volume method (FVM). In this work, two different heat flux boundary conditions are considered for the east wall: a uniform and a sinusoidally varying heat flux profile. The results show that as the value of conduction-radiation decreases, the dimensionless temperature in the medium increases. Also, it is clarified that, for an arbitrary value of the conduction-radiation parameter, the temperature decreases with decreasing scattering albedo. It is observed that when the boundaries reflect more, a higher temperature is achieved in the medium and on boundaries.
\end{abstract}

Keywords. Conduction-radiation heat transfer; transient, surface radiation; lattice Boltzmann method; finite volume method; sinusoidal heating boundary.

\section{Introduction}

Lattice Boltzmann method (LBM) has been introduced as an efficient numerical method for simulating fluid flows and heat transfer in fluids, recently [1-5]. LBM has lots of advantages such as providing a physical meaning of a problem, simple computer implementation as well as parallel programming, easy employing for complicated geometries and boundary conditions and accurate results [2, 6, 7]. Combined conduction and radiation heat transfer problems have been used in a variety of practical applications [8]. Among them, the flux boundary condition problems have been attended to in important fields of research such as the furnace design, solidification-melting of semitransparent materials, fire protection systems, porous materials, glass fabrication, electronic devises and power plants [9]. In the literature, combined radiation-conduction heat transfer problems with constant temperature boundary conditions have been studied significantly; however, a few studies have presented heat transfer problems with heat flux boundary conditions. Ho et al [10] used LBM to solve a non-Fourier conduction heat transfer problem in a planar medium. They developed the lattice Boltzmann equation for governing equations of the problem. They simulated both finite and semi-infinite computational

*For correspondence domains, which were subject to the linear and nonlinear boundary conditions. Jiaung et al [11] studied heat conduction with two types of prescribed heat flux and temperature in a planar medium using LBM. In engineering, the first application of the discrete ordinates method (DOM) was reported by Fiveland [12] in 1984. He used DOM to solve the radiative transport equation in a 2D square cavity with low temperature boundary condition containing isothermal absorbing-emitting medium. Finite-volume method (FVM) is widely employed for calculating the radiative quantities in radiative heat transfer problems [4, 13-17]. The computational grids in FVM for the radiative heat transfer are correspondent with the FVM grids that are utilized in the solution of the momentum and energy equations [18]. Furthermore, in FVM, the ray effect is considerably less due to fully conservative form of the equations [19-22]. Recently, problems of conduction-radiation heat transfer were solved using FVM coupled with LBM [23, 24]. For a 1D planar absorbing, emitting and scattering medium, Mishra and Lankadasu [25] showed that LBM shows a good accuracy of the results with higher computational time than the FDM/FVM with and without considering radiation. Mondal and Mishra [6, 26, 27] studied thermal radiation in three geometries of 1D planar, 2D square and 3D cubical using LBM. They considered both types of constant temperature and heat flux boundary conditions. Mishra and Sahai [23] and Mishra et al [28] presented the computational 
advantage of LBM in conduction-radiation heat transfer problems for solving the energy equation. They also showed the ability of FVM in computing the volumetric radiative heat transfer through the energy equation. Mondal and Chatterjee [29] performed a numerical study by LBM to solve transient heat conduction problems with and without volumetric heat generation-absorption in 2D and 3D Cartesian geometries. Uniform lattices were considered for both geometries. For both geometries one of the walls was heated and cooled with a sinusoidal function and the rest of the walls were cooled isothermally. Effects of amplitude of the sinusoidal function and volumetric heat generation-absorption on temperature profiles were analysed. Sun and Zhang [24, 30] studied the combined conduction-radiation heat transfer problems in 1D planar and 2D square enclosure using both LBM and FVM; however, they employed the same grid systems for both LBM and FVM using a relatively new boundary treatment for the thermal LBM. Sasmal and Mishra [31] studied the performance of different types of discrete heated regions along the south boundary in a 2D square enclosure using different methods of DOM: the conventional discrete ordinate method (CDOM) and the FVM. They showed that from the computational point of view, all the methods are equally efficient.

According to the literature review, all of the aforementioned conduction-radiation heat transfer problems employed constant temperature alone or combined with heat flux boundary conditions for the boundaries. However, no attempt in the literature has been made so far to solve conduction-radiation heat transfer problems involving different heat fluxes on all boundary conditions using LBM. The novelty of this paper is to study transient conductionradiation heat transfer in a $2 \mathrm{D}$ rectangular cavity with two different types of heat fluxes on the right boundary. In the first case, all of the boundaries are at constant prescribed fluxes; however, the flux on the right wall is higher than the other walls. In the second one, the right wall is heated with a sinusoidal function and the other walls are heated with uniform and similar fluxes. The medium is considered absorbing, emitting and scattering. For the medium, LBM is employed to solve the energy equation and FVM is used to solve the radiative heat transfer equation. The effects of different parameters, i.e., the scattering albedo, the conduction-radiation parameter and the extinction coefficient under consideration of different types of heat fluxes on the right boundary are investigated.

\section{Mathematical modelling}

\subsection{Model description}

A schematic of the geometry as well as the boundary conditions is shown in figure 1. The walls are assumed to be diffuse and grey. Furthermore, the contained medium is grey, absorbing, emitting, anisotropically scattering and homogeneous. The system is initially at the temperature

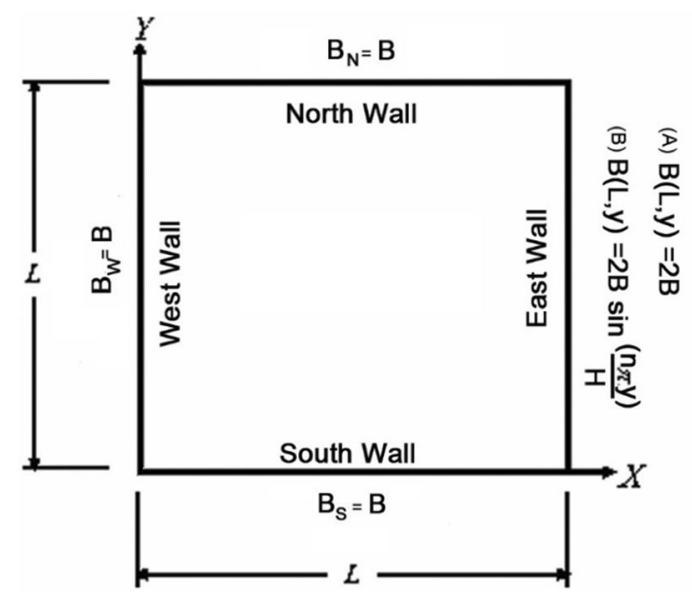

Figure 1. Schematic geometry of the problem.

$T_{\text {ref }}$ and then different heat fluxes are applied to the system from the boundaries. Two different cases are studied for the boundaries. In the first case, all of the walls have uniform fluxes; however, the east wall has a higher heat flux than the others. For the second case, a sinusoidally varying heat flux profile is applied on the east wall. The rest of the boundaries for both the cases have uniform and similar fluxes. It should be noted that all thermo-physical and optical properties of the gas are assumed to be constant. Note that $\varepsilon$ is the emissivity of the wall and the extinction coefficient, the scattering albedo and the conduction-radiation parameter are $\beta, \omega$ and $N$, respectively. It should be noted that in all the studied cases, $B$ is considered to be $500 \mathrm{~W} / \mathrm{m}^{2}$.

\subsection{Governing equations}

The energy equation for a 2D Cartesian geometry in the presence of volumetric radiation, which is computed by LBM in this study, is given as

$$
\frac{\partial T}{\partial t}=\frac{k}{\rho c_{p}}\left(\frac{\partial^{2} T}{\partial x^{2}}+\frac{\partial^{2} T}{\partial y^{2}}\right)-\nabla \cdot q_{r}
$$

where $k$ is the thermal conductivity, $\rho$ is the density, $c_{p}$ is the specific heat and $q_{r}$ is the radiative heat flux. $\nabla . q_{r}$ is the divergence of radiative heat flux, which is calculated by FVM and is given as

$$
\nabla \cdot q_{r}=(1-\omega) \beta\left[4 \pi\left(\frac{\sigma T^{4}}{\pi}-G\right)\right]
$$

where $G$ is the incident radiation.

\subsection{LBM for computation of thermal field}

The thermal lattice Boltzmann equation adopting a uniform lattice with BGK collision model is given as [32] 


$$
\begin{aligned}
& \frac{\partial g_{i}(\vec{r}, t)}{\partial t}+\vec{e}_{i} . \nabla g_{i}(\vec{r}, t)=-\frac{1}{\tau}\left[g_{i}(\vec{r}, t)-g_{i}^{e q}(\vec{r}, t)\right] \\
& i=0,1,2,3, \ldots, b .
\end{aligned}
$$

After discretization [32]

$$
g_{i}\left(\vec{r}+\vec{e}_{i} \Delta t, t+\Delta t\right)-g_{i}(\vec{r}, t)=-\frac{1}{\tau}\left[g_{i}(\vec{r}, t)-g_{i}^{e q}(\vec{r}, t)\right] .
$$

Equation (4) is the LB equation, which describes the evolution of the particle distribution function $g_{i}$ and shows the transient heat conduction problem; $g_{i}$ is the energy particle distribution function denoting the number of particles at the lattice node [6]; $b$ is the number of directions through which the information propagates and $(b+1)$ is the number of particle distribution functions in a lattice node; $g_{i}^{(e q)}$ is the equilibrium distribution function.

For conduction heat transfer problems, D2Q9 is usually used for two-dimensional problems [24]. The relaxation time $\tau$ for the D2Q9 lattices is given as follows:

$$
\tau=\frac{3 \alpha}{\left|\vec{e}_{i}\right|^{2}}+\frac{\Delta t}{2} .
$$

The discrete velocities in the D2Q9 lattice are given as follows:

$$
\begin{aligned}
\vec{e}_{i} & =(0.0) C \quad i=0 \\
\vec{e}_{i} & =C\left(\cos \frac{\pi(i-1)}{2}, \sin \frac{\pi(i-1)}{2}\right) \quad i=1,2,3,4 \\
\vec{e}_{i} & =\sqrt{2} C\left(\cos \frac{\pi(2 i-9)}{2}, \sin \frac{\pi(2 i-9)}{2}\right) \quad i=5,6,7,8
\end{aligned}
$$

where $C$ is the lattice speed defined as follows:

$$
C=\frac{\Delta x}{\Delta t}=\frac{\Delta y}{\Delta t}
$$

where $\Delta x$ and $\Delta y$ are the grid spacing in $x$ and $y$ directions, respectively, and $\Delta t$ is the discrete time step. For calculating the volumetric radiation in LBM, Eq. (4) gets modified to

$$
\begin{aligned}
g_{i}\left(\vec{r}+\vec{e}_{i} \Delta t, t+\Delta t\right)= & g_{i}(\vec{r}, t)-\frac{\Delta t}{\tau}\left[g_{i}(\vec{r}, t)-g_{i}^{e q}(\vec{r}, t)\right] \\
& -\left(\frac{\Delta t \omega_{i}}{\rho c_{p}}\right) \nabla \cdot q_{r} .
\end{aligned}
$$

Equation (8) describes a transient conduction-radiation heat transfer problem studied in this study. Then, the temperature is calculated by summing $g_{i}$ over all directions as follows:

$$
T(\vec{r}, t)=\sum_{i=0}^{8} g_{i}(\vec{r}, t) .
$$

For heat conduction and heat transfer problems, the equilibrium distribution function is given as

$$
g_{i}^{e q}(\vec{r}, t)=\omega_{i} T(\vec{r}, t)
$$

It should be noted that in this equation, the summation of the weighting factors should be equal to 1 . Therefore, from Eqs. (9) and (10)

$$
\sum_{i=0}^{8} g_{i}^{e q}(\vec{r}, t)=\sum_{i=0}^{8} \omega_{i} T(\vec{r}, t)=T(\vec{r}, t)=\sum_{i=0}^{8} g_{i}(\vec{r}, t) .
$$

The governing equations can be non-dimensionalized by the following dimensionless parameters:

$$
\begin{aligned}
r^{+} & =\frac{r}{L_{r e f}}, \theta=\frac{T}{T_{r e f}}, N=\frac{k \beta}{4 \sigma T_{r e f}^{3}}, G^{+}=\frac{G}{\left(\sigma T_{r e f}^{4} / \pi\right)}, \xi \\
& =\alpha \beta^{2} t .
\end{aligned}
$$

Therefore, the non-dimensional form of the energy equation can be given as

$$
\begin{aligned}
g_{i}^{+}\left(\vec{r}^{+}+\vec{e}_{i}^{+} \Delta \xi, \xi+\Delta \xi\right)= & g_{i}^{+}\left(\vec{r}^{+}, \xi\right) \\
& -\frac{\Delta \xi}{\tau^{+}}\left[g_{i}^{+}\left(\vec{r}^{+}, \xi\right)-g_{i}^{+(e q)}\left(\vec{r}^{+}, \xi\right)\right] \\
& -(4 N) \nabla \cdot q_{r}^{+}
\end{aligned}
$$

where $g_{i}^{+}$is the non-dimensional form of $g_{i}$ and it is obtained from the non-dimensional temperature. $\nabla \cdot q_{r}^{+}$is given as follows:

$$
\nabla \cdot q_{r}^{+}=4(1-\omega)\left[\theta^{4}-\frac{G^{+}}{4 \pi}\right]
$$

and the non-dimensional relaxation time $\tau^{+}$is given as follows:

$$
\tau^{+}=\frac{3 \alpha}{\left|\nabla r^{+} / \nabla \xi\right|^{2}}+\frac{\Delta \xi}{2}
$$

where $\nabla r^{+} / \nabla \xi$ is the non-dimensional velocity.

\subsection{FVM for computation of radiative heat flux divergence}

For absorbing, emitting and anisotropically scattering medium, the radiative heat transfer in any discrete direction $s^{m}$ with direction index $m$ is given as

$$
\frac{d I^{m}}{d s^{m}}=\widehat{s}^{m} \cdot \nabla I^{m}\left(r, \widehat{s}^{m}\right)=-\beta I^{m}+S^{m}
$$

where

$$
\widehat{s}^{m}=\left(\sin \theta^{m} \cos \phi^{m}\right) \hat{i}+\left(\sin \theta^{m} \sin \phi^{m}\right) \hat{j}+\left(\cos \theta^{m}\right) \hat{k} .
$$

The source term $S$ is given as follows:

$$
S=\beta(1-\omega)\left(\frac{\sigma T^{4}}{\pi}\right)+\frac{\beta \omega}{4 \pi} G
$$


The DOM is used to turn the transfer equation into a set of simultaneous partial differential equations. DOM can be performed to any arbitrary order and accuracy such that the total solid angle of $4 \pi$ is divided into $m$ directions. The incident radiation $(G)$ in this equation and Eq. (2) is numerically computed as follows [32]:

$$
\begin{aligned}
G & =\int_{\Omega=0}^{4 \pi} I(\Omega) d \Omega=\int_{\phi=0}^{2 \pi} \int_{\theta=0}^{\pi} I(\theta, \phi) \sin \theta \cos \theta d \theta d \phi \\
& \approx \sum_{k=1}^{M_{\phi}} \sum_{l=1}^{M_{\theta}} I^{m}\left(\theta_{l}^{m}, \phi_{k}^{m}\right) 2 \sin \theta_{l}^{m} \sin \left(\Delta \theta_{l}^{m} / 2\right) \Delta \phi_{k}^{m}
\end{aligned}
$$

where $M_{\theta}$ and $M_{\phi}$ are the number of discrete points over the complete span of the polar angle $0 \leq \theta \leq \pi$ and azimuthal angle $0 \leq \phi \leq 2 \pi$, respectively, and $I^{m}$ is the intensity in the discrete direction $\Omega^{m}$ having index $m$. The radiative heat fluxes are calculated in two directions as follows [6]:

$$
\begin{gathered}
q_{r}=\int_{\Omega=0}^{4 \pi} I(\Omega) \cos \theta d \Omega=\int_{\phi=0}^{2 \pi} \int_{\theta=0}^{\pi} I(\theta, \phi) \sin \theta \cos \theta d \theta d \phi \\
q_{r, x} \approx \sum_{k=1}^{M_{\phi}} \sum_{l=1}^{M_{\theta}} I^{m}\left(\theta_{l}^{m}, \phi_{k}^{m}\right) \sin \theta_{l}^{m} \cos \left(\phi_{k}^{m}\right) \Delta \theta_{l}^{m} \Delta \phi_{k}^{m}(20-\mathrm{a}) \\
q_{r, y} \approx \sum_{k=1}^{M_{\phi}} \sum_{l=1}^{M_{\theta}} I^{m}\left(\theta_{l}^{m}, \phi_{k}^{m}\right) \cos \left(\theta_{l}^{m}\right) \sin \left(\phi_{k}^{m}\right) \Delta \theta_{l}^{m} \Delta \phi_{k}^{m}
\end{gathered}
$$

To compute $I^{m}$ in Eq. (16), the radiative heat transfer equation in FVM is resolved into $x$ and $y$ coordinate directions and $\Delta \Omega^{m}$ is integrated over the elemental solid angle and consequently

$$
\frac{\partial I^{m}}{\partial x} D_{x}^{m}+\frac{\partial I^{m}}{\partial y} D_{y}^{m}=-\beta I^{m} \Delta \Omega^{m}+S^{m} \Delta \Omega^{m}
$$

where $D^{m}$ is given as follows:

$$
D^{m}=\int_{\Delta \Omega^{m}}\left(\widehat{n} \cdot \widehat{s}^{m}\right) d \Omega
$$

$\hat{n}$ is the outward normal to a surface at any discrete direction. When $\hat{n}$ points towards $x$ or $y$ positive coordinate directions, $D_{x}^{m}$ and $D_{y}^{m}$ are given as follows:

$$
\begin{aligned}
D_{x}^{m} & =\int_{\Delta \Omega^{m}} \sin \theta \cos \phi d \Omega=D_{x}^{m}=\int_{\Delta \Omega^{m}} \sin \theta \cos \phi d \Omega \\
& =\int_{\phi^{m}-\frac{\Delta \phi}{2}}^{\phi^{m}+\frac{\Delta \phi}{2}} \int_{\theta^{m}-\frac{\Delta \theta}{2}}^{\theta^{m}+\frac{\Delta \theta}{2}} \sin ^{2} \theta \cos \phi d \theta d \phi \\
& =\cos \phi^{m} \sin \left(\Delta \phi^{m} / 2\right)\left[\Delta \theta^{m}-\cos 2 \theta^{m} \sin \left(\Delta \theta^{m}\right)\right]
\end{aligned}
$$

$$
\begin{aligned}
D_{y}^{m} & =\int_{\Delta \Omega^{m}} \sin \theta \sin \phi d \Omega=\int_{\phi^{m}-\frac{\Delta \phi}{2}}^{\phi^{m}+\frac{\Delta \phi}{2}} \int_{\theta^{m}-\frac{\Delta \theta}{2}}^{\theta^{m}+\frac{\Delta \theta}{2}} \sin ^{2} \theta \sin \phi d \theta d \phi \\
& =\sin \phi^{m} \sin \left(\Delta \phi^{m} / 2\right)\left[\Delta \theta^{m}-\cos 2 \theta^{m} \sin \left(\Delta \theta^{m}\right)\right]
\end{aligned}
$$

where $\Delta \theta^{m}$ and $\Delta \phi^{m}$ are the elemental polar and azimuthal angles, respectively, with the centre of discrete angles $\left(\theta^{m}, \phi^{m}\right)$. For $\hat{n}$ pointing towards the negative coordinate directions, signs of $D_{x}^{m}$ and $D_{y}^{m}$ are opposite to what are given from Eqs. (23-a) and (23-b). In Eq. (21), $\Delta \Omega^{m}$ is given as follows:

$$
\begin{aligned}
\Delta \Omega^{m} & =\int_{\Delta \Omega^{m}} d \Omega^{m}=\int_{\phi=0}^{2 \pi} \int_{\theta^{m}-\frac{\Delta \theta}{2}}^{\theta^{m}+\frac{\Delta \theta}{2}} \sin \theta d \theta d \phi \\
& =2 \sin \theta^{m} \sin \left(\Delta \theta^{m} / 2\right) \Delta \phi^{m} .
\end{aligned}
$$

Integrating Eq. (16) gives

$$
\left[I_{E}^{m}-I_{W}^{m}\right] A_{x} D_{x}^{m}+\left[I_{N}^{m}-I_{S}^{m}\right] A_{y} D_{y}^{m}=\left[-\beta V I_{p}^{m}+V S_{p}^{m}\right] \Delta \Omega^{m}
$$

where $A_{x}$ and $A_{y}$ are the areas of the 2D control volume in $x$ and $y$ faces, respectively. In Eq. (25), $I$ with suffixes $E, W$, $N$ and $S$ are defined as the average intensities at the east, west, north and south control surface, respectively. Furthermore, $V$ is the volume of the cell defined as $d x \times d y$ and $I_{p}^{m}$ and $S_{p}^{m}$ are the intensities and source terms at the cellcentre $P$, respectively. In any discrete direction $\Omega^{m}$, the two cell-surface intensities and the cell-centre intensity $I_{p}^{m}$ can be obtained as follows:

$$
I_{p}^{m}=\frac{I_{E}^{m}+I_{W}^{m}}{2}=\frac{I_{N}^{m}+I_{S}^{m}}{2} .
$$

While marching from the first quadrant of a $2 \mathrm{D}$ enclosure, for which $D_{x}$ and $D_{y}$ are both positive, $I_{p}^{m}$ in terms of known $I_{W}^{m}, I_{S}^{m}$ and $S_{p}^{m}$ is given from Eqs. (24) and (25) as

$$
I_{p}^{m}=\frac{2 D_{x}^{m} A_{x} I_{W}^{m}+2 D_{y}^{m} A_{y} I_{S}^{m}+(V \Delta \Omega) S_{p}^{m}}{2 D_{x}^{m} A_{x}+2 D_{y}^{m} A_{y}+(\beta V \Delta \Omega)} \quad 1^{\text {st }} \text { quadrant. }
$$

For the other quadrants, $I_{p}^{m}$ can be obtained as follows:

$$
\begin{gathered}
I_{p}^{m}=\frac{2\left|D_{x}^{m}\right| A_{x} I_{E}^{m}+2 D_{y}^{m} A_{y} I_{S}^{m}+(V \Delta \Omega) S_{p}^{m}}{2\left|D_{x}^{m}\right| A_{x}+2 D_{y}^{m} A_{y}+(\beta V \Delta \Omega)} \quad 2^{\text {nd }} \text { quadrant, } \\
I_{p}^{m}=\frac{2\left|D_{x}^{m}\right| A_{x} I_{E}^{m}+2\left|D_{y}^{m}\right| A_{y} I_{N}^{m}+(V \Delta \Omega) S_{p}^{m}}{2\left|D_{x}^{m}\right| A_{x}+2\left|D_{y}^{m}\right| A_{y}+(\beta V \Delta \Omega)} \quad 3^{\text {th }} \text { quadrant, }
\end{gathered}
$$




$$
I_{p}^{m}=\frac{2 D_{x}^{m} A_{x} I_{W}^{m}+2\left|D_{y}^{m}\right| A_{y} I_{N}^{m}+(V \Delta \Omega) S_{p}^{m}}{2 D_{x}^{m} A_{x}+2\left|D_{y}^{m}\right| A_{y}+(\beta V \Delta \Omega)} \quad 4^{\text {th }} \text { quadrant. }
$$

For marching from any of the corners, the boundary intensity should be obtained for solving Eq. (25). Therefore, for a diffuse-grey wall, the boundary intensity $I_{b}$ at the east and west boundaries are calculated [6]:

$$
\begin{aligned}
I_{b}= & \frac{\varepsilon_{b} \sigma T_{b}^{4}}{\pi}+\left(\frac{1-\varepsilon_{b}}{\pi}\right) \sum_{k=1}^{M_{\phi}} \sum_{l=1}^{M_{\theta} / 2} I^{m}\left(\theta_{l}^{m}, \phi_{k}^{m}\right) \\
& \times \sin \left(\theta_{l}^{m}\right) \cos \left(\theta_{l}^{m}\right) \Delta \theta_{l}^{m} \Delta \phi_{k}^{m}
\end{aligned}
$$

and for the north and south boundaries

$$
\begin{aligned}
I_{b} & =\frac{\varepsilon_{b} \sigma T_{b}^{4}}{\pi}+\left(\frac{1-\varepsilon_{b}}{\pi}\right) \\
& \sum_{k=1}^{M_{\phi}} \sum_{l=1}^{M_{\theta} / 2} I^{m}\left(\theta_{l}^{m}, \phi_{k}^{m}\right) \cos \left(\theta_{l}^{m}\right) \sin \left(\theta_{l}^{m}\right) \Delta \theta_{l}^{m} \Delta \phi_{k}^{m} .
\end{aligned}
$$

\subsection{Heat flux boundary condition}

The procedure for the implementation of heat flux boundary condition in the LBM is presented in [6]. In the presence of volumetric radiation, the total heat flux on a wall results in conductive and radiative heat fluxes. The divergence of radiative heat flux $\left(\nabla \cdot q_{r}\right)$ is calculated with initial and boundary conditions. In the DOM and also in the LBM, the 2D solution space is first discretized. It is to be noted that in the LBM, for the boundary lattices, lattice centres lie along the boundaries. Thus, they stretch half distance beyond the respective boundaries. Therefore, if $N_{x} N_{y}$ is the number of control volumes in the DOM, the LBM will have $\left(N_{x}+\right.$ 1) $\left(N_{y}+1\right)$ lattices [26, 27]. In the LBM, $\nabla \cdot q_{r}$ are required at lattice centres, whereas in the DOM they are calculated at the centres of the DOM control volumes. Therefore, the average of the $\nabla \cdot q_{r}$ values should be calculated at four centres of the DOM control volumes. All boundary intensities incident at any point contribute to the radiative heat flux.

In implementing the flux boundary condition in the LBM, conservation of energy is applied to the half-size boundary lattice. This conservation of energy over time $\Delta t$ to the half-size lattice at the east boundary $i=1$ yields is given as follows [6]:

$$
\rho c_{p} \int_{0}^{\Delta x / 2}\left(\int_{t}^{t+\Delta t} \frac{\partial T}{\partial t} d t\right) d x=\int_{t}^{t+\Delta t}\left(q_{\text {in }}-q_{\text {out }}\right) d t
$$

where $q_{i n}$ is the total heat flux at the east boundary and $q_{\text {out }}=q_{c}+q_{r}$ is the heat flux leaving the half-size of the boundary lattice. Using Fourier's law for $q_{c}$, Eq. (29) is discretized to [6]

$\rho c_{p}\left(T_{i}^{n+1}-T_{i}^{n}\right) \frac{\Delta x}{2}=\int_{t}^{t+\Delta t}\left(\left(q_{i n}-q_{r}\right)-k\left[\frac{T_{i}^{n}-T_{i}^{n+1}}{\Delta x}\right]\right) d t$

where $n$ and $n+1$ represent the time levels at $t$ and $t+\Delta t$, respectively. Using the explicit scheme for the time discretization on the right-hand side of Eq. (30) gives [6]

$$
T_{i}^{n+1}=(1-\lambda) T_{i}^{n}+\lambda T_{i+1}^{n}+\eta\left(q_{i n}-q_{r}\right)
$$

where $\lambda=2 \alpha \Delta t /(\Delta x)^{2}$ and $\eta=2 \Delta t / \rho c_{p} \Delta x$. Once temperature $T_{i}^{n+1}$ at the boundary is known in terms of prescribed flux $q_{i n}, g_{i}$ at any point of the boundary can be calculated from reference [25] for the constant boundary condition.

For heat flux boundary condition, temperature remains an unknown quantity at the wall, which will be corrected trough the iterations. Therefore, from Eq. (31), $T_{i}^{n+1}$ keeps on updating with iterations, which depends on the temperature distribution of the entire domain and radiative heat flux $q_{r}$ at the boundary. Later, after the calculation of $\nabla . q_{r}$ in the LBM, the equilibrium particle distribution function $g_{i}^{(e q)}$ from Eq. (10) is calculated in the first iteration. Next, the new particle distribution functions $g_{i}\left(\vec{r}+\vec{e}_{i} \Delta t, t+\Delta t\right)$ are calculated from Eq. (8) and then they are propagated to neighbouring lattices. Finally, the new temperature is computed from Eq. (9). If the convergence is not achieved, to satisfy the boundary conditions, the particle distribution functions are locally modified and the new particle distribution functions are calculated. With the new temperature field, an updated value of $\nabla . q_{r}$ is computed. The procedure is repeated until the convergence is achieved $[6,26,27]$.

\subsection{Solution strategy}

In the 2D computational domain, the equations of LBM and FVM should be solved to calculate the values of dependent variables $\nabla . q_{r}$ and $T$ at each nodal point. As mentioned, LBM form of the energy equation is obtained using BGK approximation. FVM is used to solve the radiative equation. For this purpose, the solution domain is divided into a finite number of lattices and control volumes. For studying the grid independence, several uniform grids for the lattices in LBM and control volumes in FVM are tested. For solving the energy equation in LBM, $\nabla . q_{r}$ should be obtained at the lattice centres. However, in FVM, at the mid-points of the control surfaces and at the centre of the control volume, the intensity distributions are calculated. Therefore, $\nabla . q_{r}$ value at any lattice centre is computed by averaging $\nabla \cdot q_{r}$ values at control surfaces surrounding the lattice centre. 


\section{Results and discussion}

For the code validation, the results were compared to those obtained in reference [6] for the same conditions in a $2 \mathrm{D}$ square medium at $\xi=0.03$. The medium is grey, absorbing, emitting, anisotropically scattering and homogeneous. The media is initially at temperature $T_{\text {ref }}$. All boundaries are considered to be black. The south and north boundaries are subjected to heat fluxes $B_{S}$ and $B_{N}$, respectively, and the rest of the two boundaries remain at the constant temperature $T_{r e f}$. Figure 2 shows the variation of dimensionless temperature $\theta$ on the horizontal centreline for $\beta=1, \omega=0$ and $N=0.01$. Since the boundary conditions of the opposite walls are the same, the temperature at the centreline is symmetric about the horizontal mid-line.

In the next stage, the numbers of lattices in LBM as well as number of control volumes and discrete rays in FVM should be determined to calculate the divergence of radiative heat flux. The effects of the control volumes/lattices number as well as number of directions on dimensionless temperature $\left(T / T_{\text {ref }}\right)$ at two different locations $y / H=0.5$ and 0.7 along the centreline $x / L=0.5$ is schematized in table 1. It is assumed that $\beta=1.0, \omega=0.0$ and $N=0.1$. For the number of discrete directions $\left(M_{\theta} M_{\phi}\right)$ equal to $4 \times 8$, there is a slight deviation in the temperature for the lattice size $21 \times 21$; however, for the lattices $31 \times 31$ and $41 \times 41$, there is no significant deviation. Finally, the $41 \times 41$ lattice

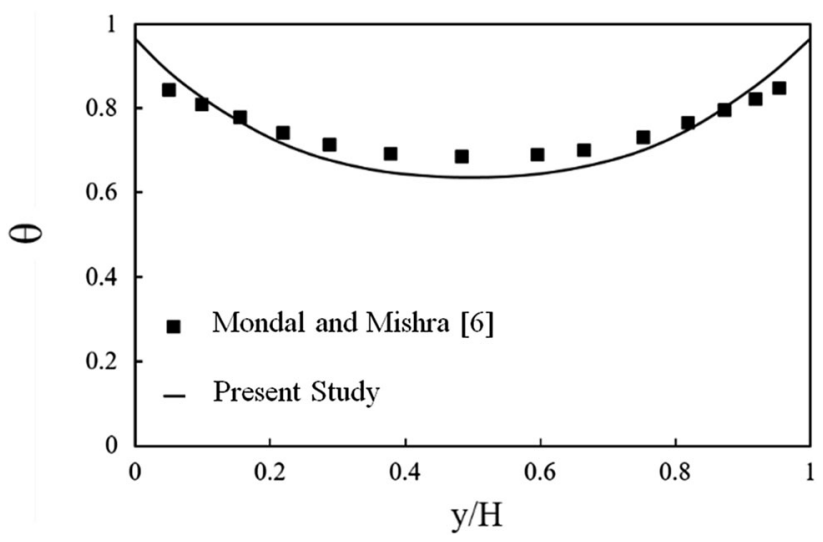

Figure 2. The variation of temperature on the horizontal centreline in compare to reference [6] for $\beta=1.0, \omega=0.0$ and $N=0.01$. size is selected for the solution of the problem. Furthermore, for $41 \times 41$ lattices, effects of the number of discrete directions on the dimensionless temperature at two different locations $y / H=0.5$ and 0.7 are also listed in table 1 . No significant change is observed beyond $4 \times 8$ rays. Therefore, all results are presented for $41 \times 41$ lattices and $4 \times 8$ rays. Note that a dimensionless time step size is $\xi=1 \times 10^{-4}$.

After studying the validation and ray independence, transient study of the 2D conduction-radiation heat transfer problem is performed using LBM with non-uniform flux boundary conditions on the walls. Figure $3 \mathrm{a}-\mathrm{d}$ shows the effect of imposed heat flux on the east wall $B_{E}$ on $\theta$ at different time instants $(\xi)$ along the horizontal line at $y /$ $H=0.25$ and 0.5 . The boundaries are black and the results are obtained for $\beta=1.0, \omega=0.7$ and $N=0.01$. Temperature profiles have been plotted at $\xi=0.005,0.03$ and 0.2 . It is observed that for $B_{E}>B$, the $\theta$ at centreline is nonsymmetric about the vertical mid-line. Furthermore, increase in time step causes a higher temperature in the entire domain. For the sinusoidally varying heat flux profile, due to non-uniform heat flux from the east wall and also a lower mean heat flux than in the first case, a lower temperature is achieved.

In the following, the influence of different effective parameters on the temperature profile will be investigated at $\xi=0.2$.

\subsection{Effect of conduction-radiation parameter on temperature distribution}

In figure 4 , temperature distributions within an absorbingemitting-scattering medium $(\omega=0.5$ and $\beta=1)$ have been shown along the centreline for different conductionradiation parameters $N=0.01,0.1$ and 1.0 for both cases. Radiation propagates at a faster speed compared with conduction heat transfer. Therefore, for lower values of $N$, the radiation dominance causes faster attainment of higher temperature in the domain. As the value of $N$ decreases, radiation is more effective on the temperature distribution obviously and therefore, for the radiation-dominated case, the dimensionless temperature in the medium is higher than that for the higher $N$ values.

Table 1. Effect of grid size and ray independence in the LBM-FVM at $x / L=0.5$ and $y / H=0.5$ and 0.7 for $\beta=1.0, \omega=0.0$ and $N=0.1$.

\begin{tabular}{|c|c|c|c|c|c|c|c|}
\hline \multirow[b]{2}{*}{ Number of directions } & \multirow[b]{2}{*}{ Number of lattices } & \multicolumn{2}{|c|}{$\begin{array}{l}\text { Dimensionless } \\
\text { temperature }\end{array}$} & \multirow[b]{2}{*}{ Number of lattices } & \multirow[b]{2}{*}{ Number of directions } & \multicolumn{2}{|c|}{$\begin{array}{l}\text { Dimensionless } \\
\text { temperature }\end{array}$} \\
\hline & & $\begin{array}{c}y / \\
H=0.5\end{array}$ & $\begin{array}{c}y / \\
H=0.7\end{array}$ & & & $\begin{array}{c}y / \\
H=0.5\end{array}$ & $\begin{array}{c}y / \\
H=0.7\end{array}$ \\
\hline \multirow[t]{3}{*}{$4 \times 8$} & $21 \times 21$ & 0.6645 & 0.5967 & $41 \times 41$ & $2 \times 4$ & 0.6612 & 0.5796 \\
\hline & $31 \times 31$ & 0.6641 & 0.5966 & & $4 \times 8$ & 0.6641 & 0.5966 \\
\hline & $41 \times 41$ & 0.6641 & 0.5966 & & $6 \times 12$ & 0.6639 & 0.5966 \\
\hline
\end{tabular}



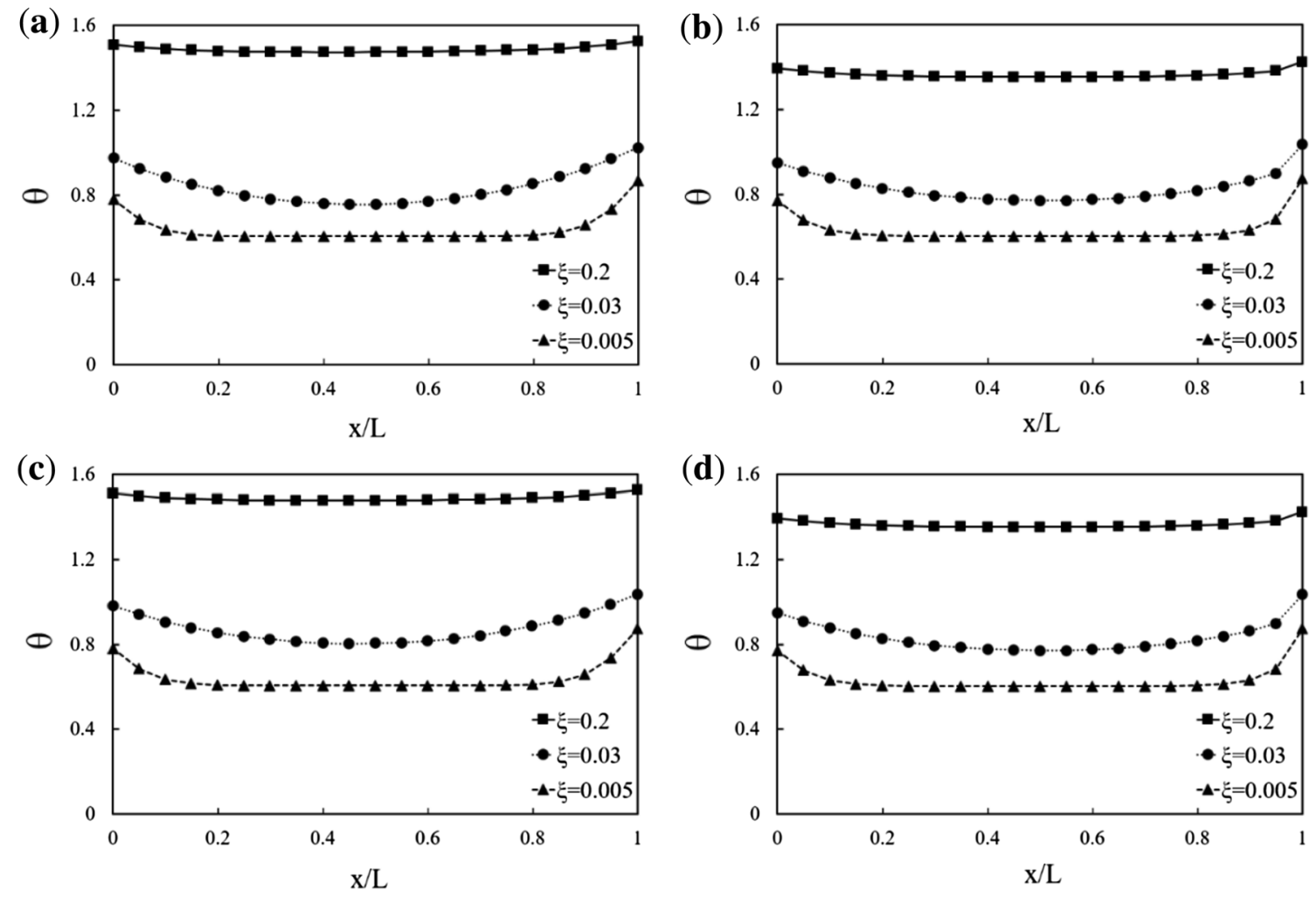

$\mathrm{B}_{\mathrm{E}}=2 \mathrm{~B}$

$\mathrm{B}_{\mathrm{E}}=2 \mathrm{~B} \sin (2 \pi \mathrm{y} / \mathrm{H})$

Figure 3. Dimensionless temperature profiles at different times along the horizontal line at $y / H=0.25$ (a, b) and 0.5 (c, d) for different heat fluxes on the right side and $\beta=1.0, \omega=0.5, N=0.01$ and $\varepsilon=1.0$.
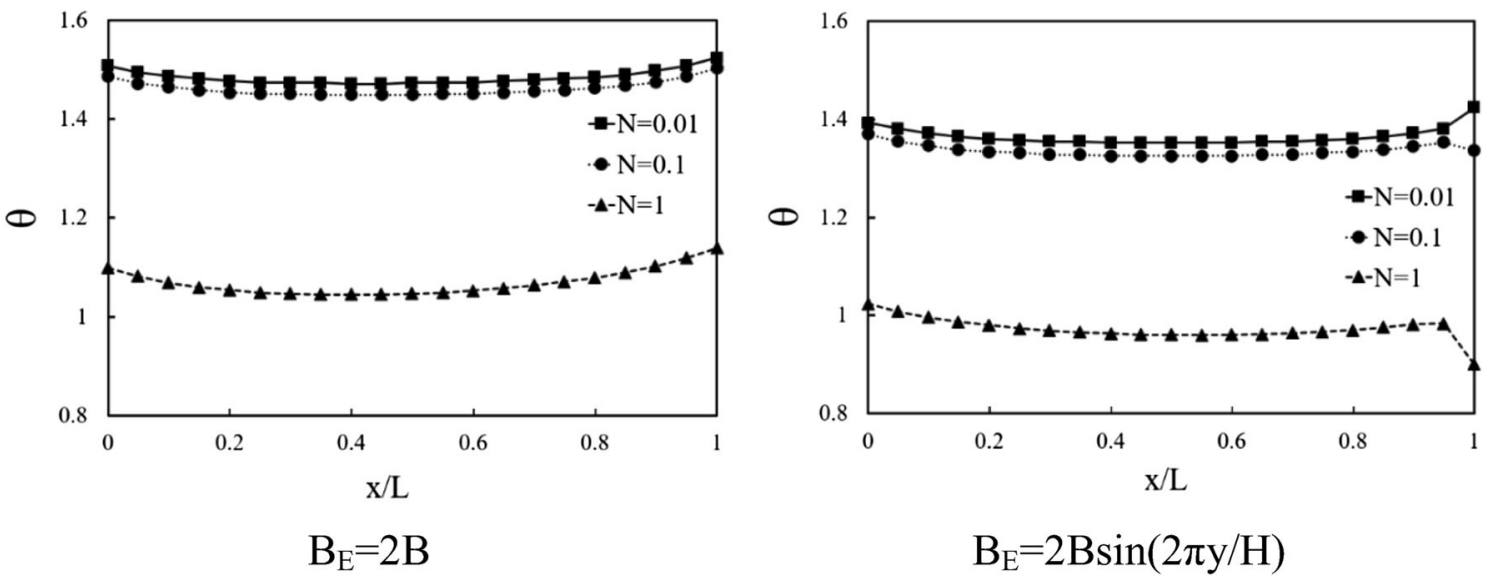

$\mathrm{B}_{\mathrm{E}}=2 \mathrm{~B} \sin (2 \pi \mathrm{y} / \mathrm{H})$

Figure 4. Comparison of the dimensionless mid-line temperature $y / H=0.5$ for different conduction-radiation parameters $\beta=1.0$, $\omega=0.5$ and $\varepsilon=1.0$

\subsection{Effect of scattering albedo on temperature distribution}

In figure 5, the variation of the dimensionless temperature for different scattering albedo values of $\omega=0.0,0.5$ and 0.9 are shown along the centreline for $\beta=1$ and $N=0.1$. The scattering albedo defines as the fraction of scattered radiation energy in the domain.
Therefore, it is obvious that the effects of the scattering albedo are more significant for lower values of $N$ $(N=0.01)$ and is very small for the conduction heat transfer dominated case $(N=1)$. Hence, for an arbitrary value of the conduction-radiation parameter, as shown in figure 5, the temperature decreases on decreasing $\omega$ since less radiation is scattered out for the lower values of scattering albedo. It is noticeable that for $\omega=1$, all radiant 


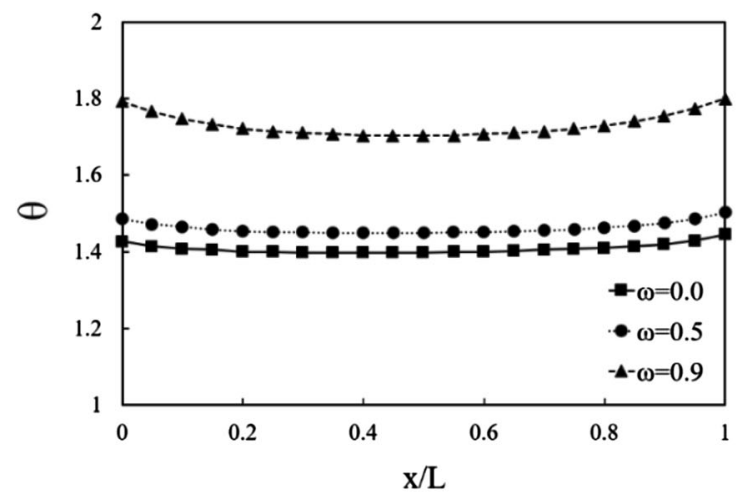

$\mathrm{B}_{\mathrm{E}}=2 \mathrm{~B}$

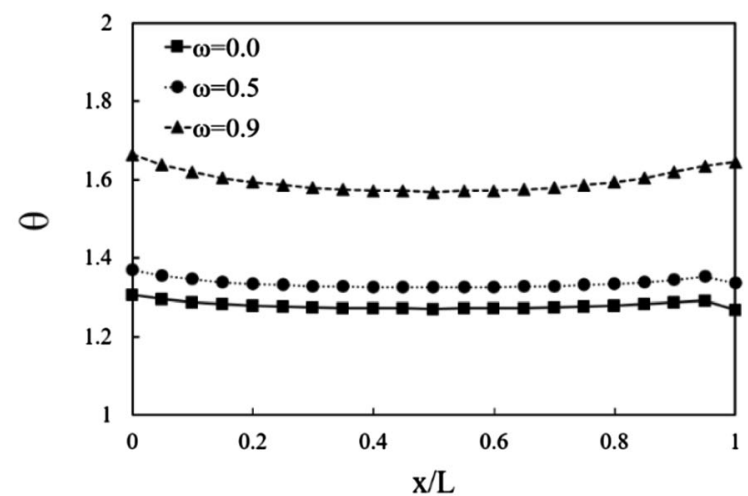

$\mathrm{B}_{\mathrm{E}}=2 \mathrm{~B} \sin (2 \pi \mathrm{y} / \mathrm{H})$

Figure 5. Comparison of dimensionless temperature at $\xi=0.2$ for variable scattering albedo radiation parameters $\beta=1.0, N=0.1$ and $\varepsilon=1.0$ at the centreline

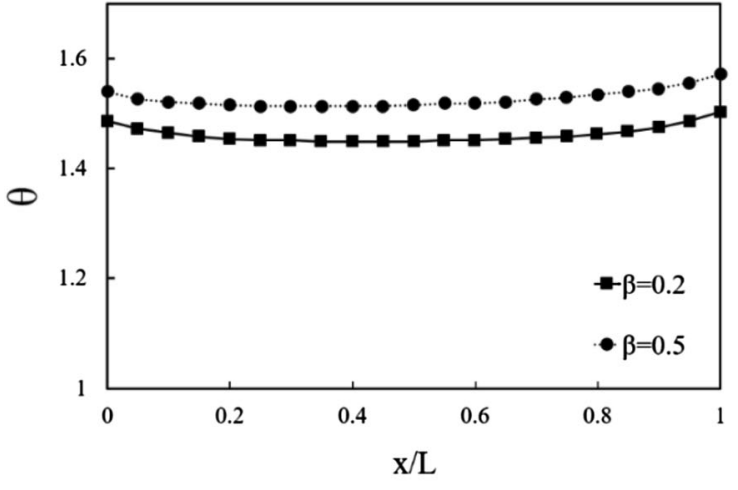

$\mathrm{B}_{\mathrm{E}}=2 \mathrm{~B}$

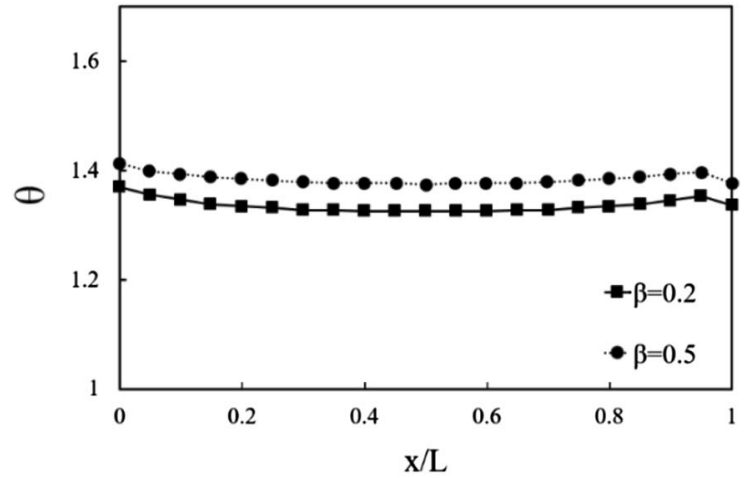

$\mathrm{B}_{\mathrm{E}}=2 \mathrm{~B} \sin (2 \pi \mathrm{y} / \mathrm{H})$

Figure 6. Comparison of non-dimensional temperature at the centreline at $\xi=0.2$ for different extinction coefficients and $\omega=0.5$, $N=0.1$ and $\varepsilon=1.0$.

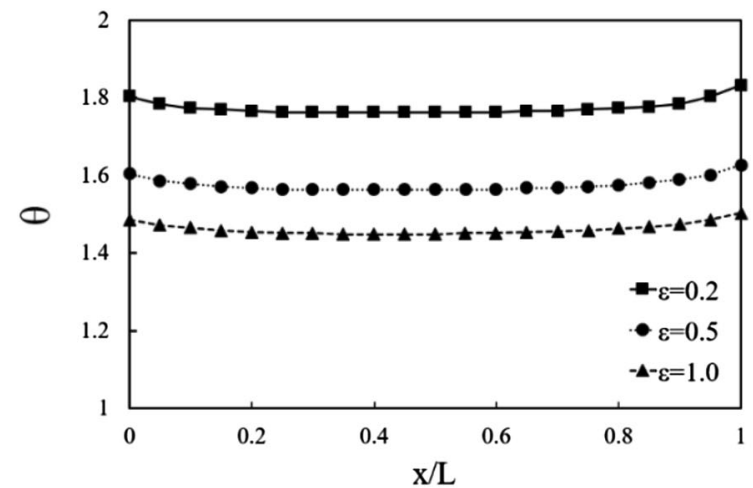

$\mathrm{B}_{\mathrm{E}}=2 \mathrm{~B}$

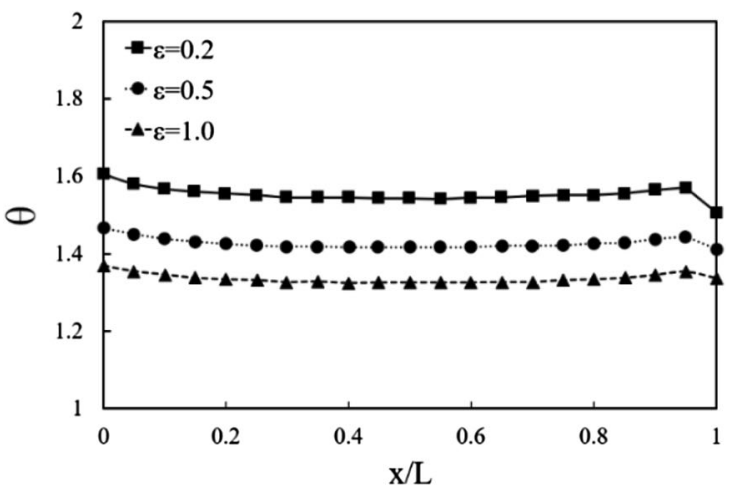

$\mathrm{B}_{\mathrm{E}}=2 \mathrm{~B} \sin (2 \pi \mathrm{y} / \mathrm{H})$

Figure 7. Comparison of non-dimensional mid-line temperature for both studied cases for different emissivity coefficients and $\beta=1.0$, $N=0.1$ and $\omega=0.5$ at $\xi=0.2$ 
energy will be scattered and therefore the problem will be only a conduction heat transfer type.

\subsection{Effect of extinction coefficient on temperature distribution}

Figure 6 shows the effect of extinction coefficient parameter $(\beta)$ on the dimensionless temperature distributions for $\omega=0.5$ and $N=0.1$ for both cases studied. It can be seen that the dimensionless temperature is increased on increasing $\beta$ since that the medium becomes more participating and due to the dominance of the radiation, a higher temperature is achieved.

\subsection{Effect of emissivity coefficient on temperature distribution}

For a completely reflecting surface $\varepsilon=0.0$, any incident radiation cannot be retained at the surface and it gives back all radiations to the medium. However, a surface with $\varepsilon=1.0$ absorbs all the incident radiations on it and it has the maximum emission corresponding to its temperature. For $\omega=0.5, N=0.1$ and $\beta=1$ in figure 7 , the dimensionless temperature distributions are shown for different emissivity coefficients for both studied cases at $\xi=0.2$. It is observed that when the boundaries reflect more, a higher temperature is achieved in the medium and boundaries.

\section{Conclusion}

The aim of this paper is to employ LBM in order to solve the energy equation of transient combined conduction-radiation heat transfer in a 2D cavity for calculating the thermal distribution in the medium. The DOM was also employed for calculating the radiative heat fluxes by FVM. Two different heat fluxes were applied to the east wall and a constant heat flux is applied to all the other walls. The effects of various parameters such as the conduction-radiation parameter, the extinction coefficient and the scattering albedo of the grey medium on the dimensionless temperature were investigated. The results show the following:

- as the value of conduction-radiation decreases, the dimensionless temperature in the medium is higher than that for the higher conduction-radiation values;

- for an arbitrary value of the conduction-radiation parameter, the temperature decreases with decreasing scattering albedo;

- it can be seen that the dimensionless temperature is increased with increasing $\beta$ since the medium becomes more participating and due to the dominance of the radiation, a higher temperature is achieved;
- it is observed that when the boundaries are more reflecting, a higher temperature is achieved in the medium and boundaries.

\section{References}

[1] Chen S and Doolen G D 1998 Latice Boltzmann method for fluid flows. Ann. Rev. Fluid Mech. 30(1): 329-364

[2] Succi S 2001 The lattice Boltzmann equation: for fluid dynamics and beyond. UK: Clarendon Press

[3] Yi H L, Yao F J and Tan H P 2016 Lattice Boltzmann model for a steady radiative transfer equation. Phys. Rev. E 90(2): 023312

[4] Keshtkar M M and Amiri B 2018 Numerical simulation of radiative-conductive heat transfer in an enclosure with an isotherm obstacle. Heat Transf. Eng. 39(1): 72-83

[5] Mondal B and Chatterjee D 2012 Lattice Boltzmann simulation of heat conduction problems in non-isothermally heated enclosures. Heat Transf. Asian Res. 31(2): 127-144

[6] Mondal B and Mishra S C 2009 The lattice Boltzmann method and the finite volume method applied to conductionradiation problems with heat flux boundary conditions. Int. J. Numer. Methods Eng. 78(2): 172-195

[7] Sajjadi H and Kefayati G R MHD turbulent and laminar natural convection in a square cavity utilizing Lattice Boltzmann Method. Heat Transf. Asian Res. 45(8): 795-814

[8] Baghban M, Mansouri S H and Shams Z 2014 Inverse radiation-conduction estimation of temperature-dependent emissivity using a combined method of genetic algorithm and conjugate gradient. J. Mech. Sci. Technol. 28(2): 739-745

[9] Siegel R 2001 Thermal radiation heat transfer, 4th edtion. USA: Taylor \& Francis

[10] Ho J R, Kuo C P, Jiaung W S and Twu C J 2002 Lattice Boltzmann scheme for hyperbolic heat conduction equation. Numer. Heat Transf. Part B Fundam. 41(6): 591-607

[11] Jiaung W S, Ho J R and Kuo C P 2001 Lattice Boltzmann method for the heat conduction problem with phase change. Numer. Heat Transf. Part B Fundam. 39(2): 167-187

[12] Fiveland W 1984 Discrete-ordinates solutions of the radiative transport equation for rectangular enclosures. J. Heat Transf. 106(4): 699-706

[13] Truelove J 1988 Three-dimensional radiation in absorbingemitting-scattering media using the discrete-ordinates approximation. J. Quant. Spectrosc. Radiat. Transf. 39(1): 27-31

[14] Fiveland W 1988 Three-dimensional radiative heat-transfer solutions by the discrete-ordinates method. J. Thermophys. Heat Transf. 2(4): 309-316

[15] Keshtkar M M, Gandjalikhan Nassab S A and Nasr M R J 2009 Heat transfer characteristics of a cylindrical porous radiant air heater under the influence of a two-dimensional axisymmetric radiative field. Proc. Inst. Mech. Eng. Part A J. Power Energy 223(8): 913-923

[16] Keshtkar M M and Gandjalikhan Nassab S A 2009 Theoretical analysis of porous radiant burners under $2 \mathrm{D}$ radiation field using discrete ordinates method. J. Quant. Spectrosc. Radiat. Transf. 110(17): 1894-1907 
[17] Ansari A and Gandjalikhan Nassab S A 2013 Forced convection of radiating gas over an inclined backward facing step using the blocked-off method. Therm. Sci. 17(3): 773-786

[18] Murakami S and Asako Y 2013 A finite volume method on distorted quadrilateral meshes for discretization of the energy equation's conduction term. Heat Transf. Asian Res. 42(2): 163-184

[19] Sun Y, Zhang, X and Howell J R 2016 Evaluation of three different radiative transfer equation solvers for combined conduction and radiation heat transfer. J. Quant. Spectrosc. Radiat. Transf. 184: 262-273

[20] Hunter B and Guo Z 2016 Improved treatment of anisotropic scattering in radiation transfer analysis using the finite volume method. Heat Transf. Eng. 37(4): 341-350

[21] Chai J C, Lee H S and Patankar S V 1994 Finite volume method for radiation heat transfer. J. Thermophys. Heat Transf. 8(3): 419-425

[22] Asghari A, Gandjalikhan Nassab S A and Ansari A 2015 Numerical study of combined radiation and turbulent mixed convection heat transfer in a compartment containing participating media. J. Mech. 31(4): 467-480

[23] Mishra S C and Sahai H 2014 Analysis of non-Fourier conduction and volumetric radiation in a concentric spherical shell using lattice Boltzmann method and finite volume method. Int. J. Heat Mass Transf. 68: 51-66

[24] Sun Y and Zhang X 2016 Analysis of transient conduction and radiation problems using lattice Boltzmann and finite volume methods. Int. J. Heat Mass Transf. 97: 611-617
[25] Mishra S C and Lankadasu A 2005 Transient conductionradiation heat transfer in participating media using the lattice Boltzmann method and the discrete transfer method. Numer. Heat Transf. Part A Appl. 47(9): 935-954

[26] Mondal B and Mishra S C 2008 Lattice Boltzmann method applied to the solution of the energy equations of the transient conduction and radiation problems on non-uniform lattices. Int. J. Heat Mass Transf. 51(1): 68-82

[27] Mondal B and Mishra S C 2009 Analysis of 3-D conductionradiation heat transfer using the Lattice Boltzmann method. J. Thermophys. Heat Transf. 23(1): 210-216

[28] Mishra S C, Poonia H, Vernekar R R and Das A K 2014 Lattice Boltzmann method applied to radiative transport analysis in a planar participating medium. Heat Transf. Eng. 35(15): 1267-1278

[29] Mondal B and Chatterjee D 2012 Lattice Boltzmann simulation of heat conduction problems in non-isothermally heated enclosures. Heat Transf. Asian Res. 41(2):127-144

[30] Sun Y and Zhang X 2015 Analysis of transient conduction and radiation problems using the lattice Boltzmann and discrete ordinates methods. Numer. Heat Transf. Part A Appl. 68(6): 619-637

[31] Sasmal A and Mishra S C 2016 Analysis of conduction and radiation heat transfer in a differentially heated 2-D square enclosure. Heat Transf. Asian Res. 46(4): 384-408

[32] Mishra S C and Roy H K 2007 Solving transient conduction and radiation heat transfer problems using the lattice Boltzmann method and the finite volume method. J. Comput. Phys. 223(1): 89-107 